\title{
Editorial: Trabajos de Prehistoria se consolida como revista española de referencia en los indicadores de calidad de revistas científicas
}

\author{
Editorial: Trabajos de Prehistoria qualified as a Spanish journal of reference in the quality \\ indicators of scientific journals
}

\author{
Luis Rodríguez Yuntaa, Pedro Díaz-del-Río ${ }^{\mathrm{b}}$ y M. ${ }^{a}$ Isabel Martínez Navarrete ${ }^{\mathrm{b}}$
}

\section{RESUMEN}

En los últimos años Trabajos de Prehistoria ha ido mejorando de forma continua su posición en los sistemas de información nacionales e internacionales que certifican la calidad de las publicaciones científicas. En este editorial se presentan datos recogidos en las principales bases de datos y rankings de revistas.

\begin{abstract}
In recent years, Trabajos de Prehistoria has been continuously improving its position in national and international information systems that certify the quality of scientific publications. This editorial note presents data collected in the main databases and journal rankings.
\end{abstract}

Palabras clave: Trabajos de Prehistoria; evaluación de las revistas; índices de impacto.

Key words: Trabajos de Prehistoria; journals evaluation; impact index.

\section{INTRODUCCIÓN}

La revista Trabajos de Prehistoria (en adelante TP) se edita desde 1960 en versión impresa y cuenta desde 2007 con una edición electrónica paralela. Toda la colección anterior está disponible en versión digital, si bien en abierto solo se puede acceder actualmente a lo publicado desde 1988. Desde 2007 la edición electrónica se elabora y distribuye al mismo tiempo que la impresa, lo que aporta notables ventajas no solo a una mayor difusión de los textos, sino también a la propia información que se ofrece, como las figuras en color (frente a la limitación de los tonos de gris en la versión impresa) o material complementario. De forma creciente los autores remiten adjuntos en diferentes formatos que incrementan el valor de la versión disponible en la web y que puede considerarse entre las buenas prácticas en la edición científica actual (Rodríguez Yunta 2014).

$T P$ forma parte de la oferta editorial del Consejo Superior de Investigaciones Científicas (CSIC). Como organismo público de investigación compite por alcanzar un puesto de referencia con otros centros y universidades a nivel nacional, europeo e internacional. Dentro de esta política, la edición de revistas es una labor más en la que se pone en juego el prestigio institucional y constituye un servicio externo para la publicación de los trabajos de investigadores de centros públicos y privados en todo el mundo. La función de la Editorial CSIC es esencial para que una revista como $T P$ se distribuya en una plataforma web de acceso abierto y responda a los continuos retos de la transformación digital (Fig. 1). Con la instalación a finales de 2020 de la versión 3 del programa de gestión Open Journal Systems se van dando pasos en esta dirección.

La valoración de las revistas científicas se encuentra mediatizada por las diferentes iniciativas que recopilan datos, clasifican y establecen comparaciones entre ellas. Aunque los indicadores bibliométricos tienen claras limitaciones (Walters 2017) y con frecuencia se reclama que no deben utilizarse de forma simplista en la evaluación

a Unidad de Apoyo a la Edición de Revistas, Centro de Ciencias Humanas y Sociales. Consejo Superior de Investigaciones Científicas. C/ Albasanz 26-28. 28037 Madrid. Correo e.: luis.ryunta @cchs.csic.es https://orcid.org/0000-0002-8424-6205

b Instituto de Historia. Consejo Superior de Investigaciones Científicas. C/ Albasanz 26-28. 28037 Madrid. Correos e.:

PDR pedro.diazdelrio@cchs.csic.es https://orcid.org/0000-0002-4150-6185 ; MIMN isabel.martinez@cchs.csic.es https://orcid.org/0000-0002-3060-6033

Copyright: (C) 2021 CSIC. Este es un artículo de acceso abierto distribuido bajo los términos de la licencia de uso y distribución "Creative Commons Reconocimiento 4.0 Internacional” (CC BY 4.0) 


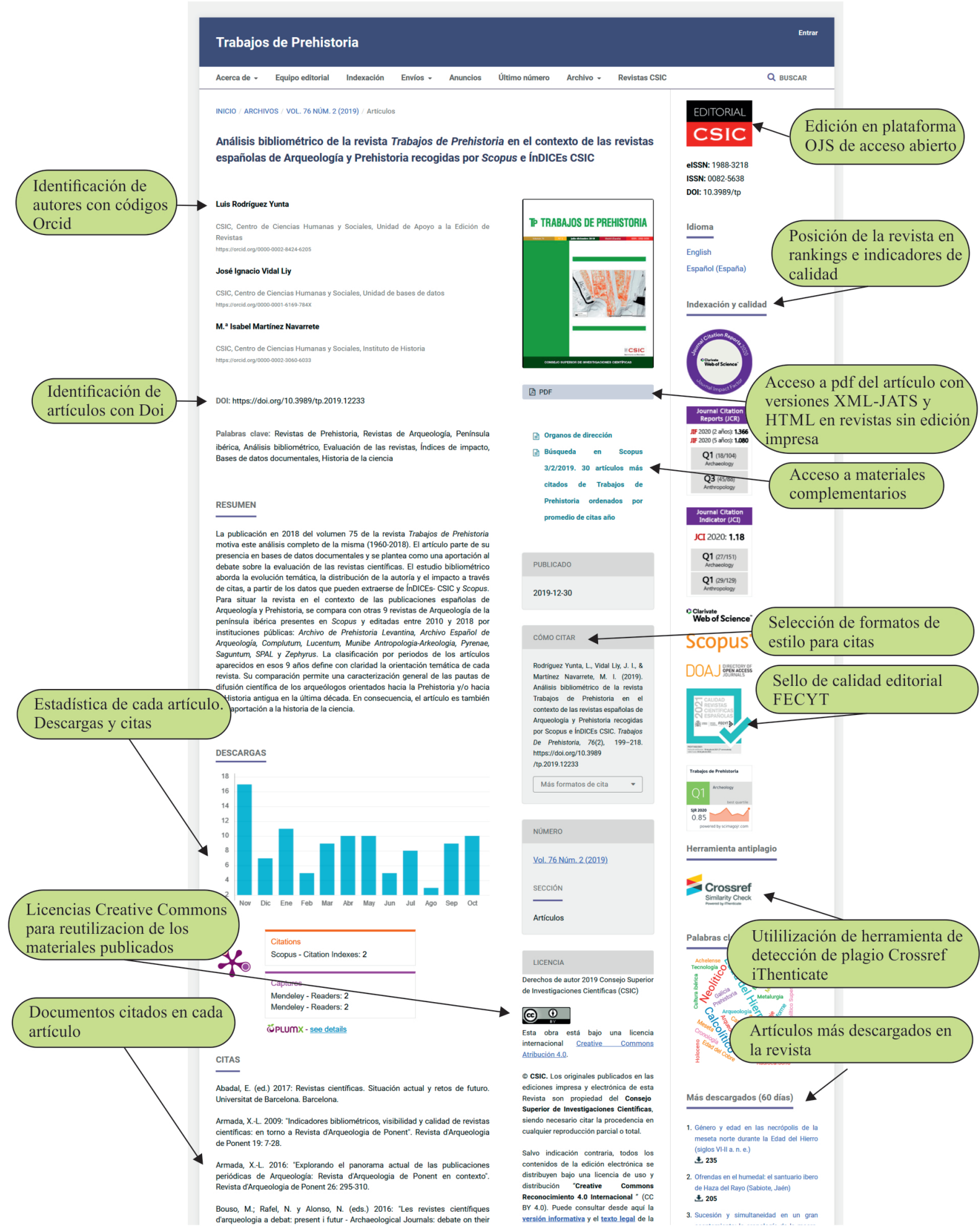

Fig. 1. Ejemplo de visualización de un artículo de Trabajos de Prehistoria y sus características técnicas en la plataforma web de acceso abierto de Editorial CSIC (consultado en octubre de 2021).

Trab. Prehist., 78, N..$^{\circ}$, julio-diciembre 2021, pp. 207-216, ISSN: 0082-5638

https://doi.org/10.3989/tp.2021.12272 
de investigadores (Cagan 2013; Hicks et al. 2015; Delgado et al. 2021), mantienen una fuerte incidencia en la política científica. Por ello, los servicios editoriales deben estar atentos a fomentar la presencia de sus publicaciones en estos recursos y analizar los resultados obtenidos para buscar el reto de una mejora continua.

Somos conscientes de que la auténtica calidad de una revista radica fundamentalmente en sus contenidos. Para ello debe darse una investigación de calidad, bien desarrollada y bien expuesta, sumado a un proceso de valoración por pares que ayude al equipo editorial a seleccionar los mejores trabajos al tiempo que aportan sugerencias de mejora de contenidos, para finalmente perfilar la parte expositiva en la preparación final para la edición. El trabajo de los comités editoriales y la aportación de expertos externos son fundamentales no solo para seleccionar los artículos de mayor interés sino también para depurar y mejorar la presentación y en definitiva la capacidad de comunicación científica.

El punto de partida de todo el proceso se inicia con la recepción de investigaciones de calidad. Esto es más probable que suceda si las publicaciones resultan atractivas para los investigadores, tanto consagrados como nóveles. El objetivo de un equipo editorial debe ser convertir una revista en una de las primeras opciones que busquen los autores para situar sus mejores trabajos. Estos deben tener la seguridad de que merece la pena todo esfuerzo para obtener el visto bueno en el proceso de revisión, porque el premio es la publicación dentro de una cabecera editorial que inspira confianza y tiene en consecuencia una adecuada difusión en el actual sistema de comunicación de la ciencia.

Los sistemas de recuperación información y buscadores académicos aportan visibilidad y mejoran la recuperabilidad de los contenidos publicados. Los artículos de TP se encuentran indexados en las principales bases de datos que incluyen el ámbito temático de esta publicación. Es una publicación fuente en los principales índices de citas, con referencias desde 2003 en Scopus (de la multinacional Elsevier) y desde 2007 en Arts \& Humanities Citation Index, A\&HCI y Social Sciences Citation Index, SSCI (actualmente producidos por la multinacional Clarivate Analytics). Entre las bases de datos especializadas internacionales, está incluida desde 1969 en Anthropological Literature (Tozzer Library, Harvard University). Los textos publicados en el periodo 1960-2000 se pueden localizar en Periodicals Index Online, PIO, y Periodicals Archive Online, PAO (productos elaborados por Chadwick-Healey que forman parte actualmente de la multinacional ProQuest). Sus artículos pueden buscarse también en los sistemas referenciales nacionales: ÍnDICES CSIC (CSIC), desde el inicio de la publicación en 1960, y Dialnet (Fundación Dialnet, Universidad de La Rioja), desde 1970. Finalmente, los metadatos de los artículos publicados que están disponibles en acceso abierto desde 1988, se integran en buscadores como REDIB, Red Iberoamericana de Innova- ción y Conocimiento Científico (CSIC y Universia) ${ }^{1}$ y DOAJ, Directory of Open Access Journals.

Los textos completos en versión electrónica están disponibles en acceso abierto para los artículos publicados desde 1988 en el sitio web: http://tp.revistas.csic.es. En el año 2021 se ha incorporado en esta plataforma la posibilidad de tener indicadores estadísticos a nivel de artículo (article-level metrics o almétricas). Los textos del periodo anterior a 1988 pueden localizarse en el recurso referido anteriormente Periodicals Archive Online.

Tras los primeros 75 volúmenes de $T P$, se publicó un análisis bibliométrico (Rodríguez Yunta et al. 2019), que se sumaba a uno anterior en el volumen 50 (Rodríguez Alcalde et al. 1993). En este editorial se actualizan y complementan los datos que se ofrecieron en dichos artículos con la aportación de las novedades introducidas en los últimos años en los indicadores de citación y los resultados obtenidos por la revista.

\section{POSICIÓN DE TRABAJOS DE PREHISTORIA EN DIRECTORIOS Y SISTEMAS DE CATEGORIZACIÓN DE REVISTAS}

La publicación se encuentra recogida por los siguientes directorios y sistemas de categorización de revistas académicas:

Latindex, Sistema Regional de Información en Línea para Revistas Científicas de América Latina, el Caribe, España y Portugal, en donde figura tanto la versión impresa como la electrónica de $T P$ en la sección de Catálogo que certifica el cumplimiento de parámetros de calidad editorial.

Relación de revistas acreditadas por el sello de calidad de la Fundación Española para la Ciencia y la Tecnología (FECYT, Ministerio de Ciencia e Innovación, Gobierno de España), que evalúa la calidad editorial y científica de las revistas españolas, obtenido por $T P$ en 2012 (tercera convocatoria) y renovado anualmente.

ERIH Plus, European Reference Index for the Humanities (Norwegian Directorate for Higher Education and Skills), que identifica las revistas científicas europeas de Humanidades y Ciencias Sociales que cumplen requisitos de calidad establecidos por la $\mathrm{Eu}$ ropean Science Foundation.

MIAR, Matriz de Información para el Análisis de Revistas (Universidad de Barcelona), que le adjudica el valor máximo de 11 en su indicador ICDS (Índice Compuesto de Difusión Secundaria, calculado sobre la visibilidad de la revista en diferentes bases de datos).

CIRC, Clasificación Integrada de Revistas Cientificas (EC3Metrics, spin-off de la Universidad de Grana-

\footnotetext{
1 REDIB (https://www.redib.org) es una plataforma de agregación de contenidos de revistas iberoamericanas de acceso abierto. Se trata de un proyecto en el que se asocia el Consejo Superior de Investigaciones Científicas y el portal Universia, la red de cooperación universitaria promovida por el Banco Santander.
} 
da), que adjudica a $T P$ la categoría principal A tanto en Humanidades como en Ciencias Sociales.

CARHUS Plus + (Gencat, Agencia de Gestión de Ayudas Universitarias y de Investigación de la Generalitat de Catalunya), que otorga a TP la categoría B en el ámbito de Historia (en donde se integran en este caso las publicaciones de Arqueología).

DOAJ, Directory of Open Access Journals, ya citado, que identifica el seguimiento de buenas prácticas en el acceso abierto a las publicaciones científicas.

\section{POSICIÓN DE TRABAJOS DE PREHISTORIA EN RANKINGS INTERNACIONALES}

Existen muchas iniciativas internacionales que aportan datos de interés sobre la difusión e impacto de las publicaciones científicas. Este análisis se centra en las dos fuentes más consolidadas y que ofrecen resultados de forma estable: los índices de citas Web of Science y Scopus ${ }^{2}$. La tabla 1 resume los datos de TP en 2020 en los principales indicadores que se elaboran a partir de estos productos y que se comentan en los apartados siguientes.

\begin{tabular}{|c|c|}
\hline $\begin{array}{c}\text { Fuente } \\
\text { de } \\
\text { datos }\end{array}$ & Indicadores y posición en rankings 2020 \\
\hline \multirow{5}{*}{$\begin{array}{l}\text { Web of } \\
\text { Science }\end{array}$} & $\begin{array}{l}\text { JIF Factor de impacto 2 años: 1,366 } \\
\text { Q1 en Arqueología (\#18/104) }\end{array}$ \\
& Q3 en Antropología (\#45/88) \\
& JCI Indicador de citación (3 años): 1,18 \\
& Q1 en Arqueología (\#27/151) \\
Q1 en Antropología (\#29/129)
\end{tabular}

Tab. 1. Resumen de datos de Trabajos de Prehistoria en los indicadores elaborados a partir de los índices de citas Web of Science y Scopus. Q1: abreviatura en inglés de primer cuartil; JIF Journal Impact Factor; JCI Journal Citation Indicator: CWTS Centre for Science and Technology Studies (Universidad de Leiden, Holanda): SNIP Source Normalized Impact per Publication, IPP Impact per Publication.

${ }^{2} \mathrm{El}$ acceso a estos recursos puede realizarse en España a partir del sitio web de la Fundación Español para la Ciencia y la Tecnología (FECYT), que gestiona la licencia nacional:

https://www.recursoscientificos.fecyt.es/

\subsection{Datos de TP en Journals Citation Reports (Web of Science)}

Clarivate Analytics ha publicado el 30 de junio de 2021 los datos correspondientes a 2020 de su informe anual Journal Citation Report (JCR) dentro de su plataforma Web of Science. Por primera vez este producto abarca las categorías de Humanidades e incluye un nuevo ranking basado en un indicador normalizado, Journal Citation Indicator (JCI). Este nuevo "indicador de citación de la revista" se basa en las citas recibidas en un año determinado a los artículos de una revista editados en los 3 años anteriores, normalizado según el valor medio de la categoría en la que se clasifica la revista. En el ranking por $J C I, T P$ se sitúa en el cuartil 1 (Q1) tanto en la agrupación de revistas de Arqueología (posición 27 de 151 revistas internacionales en esta categoría de Humanidades) como en Antropología (posición 29 de 129 títulos en esta categoría dentro de Ciencias Sociales).

$J C R$ también incluye el Journal Impact Factor (factor de impacto de la revista), que se calcula por las citas recibidas en un año a los artículos editados en los 2 años anteriores. En este ranking $T P$ se mantiene en el cuartil 3 (Q3) en Antropología (posición 45 de 88 revistas, a solo una posición de entrar en el Q2) y además se sitúa en el Q1 entre las revistas internacionales de Arqueología (posición 18 de 104 títulos).

La diferencia entre el número de títulos incluidos en las respectivas categorías temáticas del $J I F$ y del $J C I$ se explica porque el segundo ranking incorpora las publicaciones de la base de datos Emerging Source Citation Index (ESCI). Este fichero se compone de títulos que se postulan para ser incluidos en los índices de citas Science Citation Index (SCI), Social Science Citation Index (SSCI) o Arts \& Humanities Citation Index (AHCI), y cuyas citas sí se tienen en cuenta para el cálculo de los indicadores bibliométricos.

Para años anteriores $J C R$ publica sus indicadores solamente para revistas de las bases de datos SCI y SSCI, pero no para $A H C I$. Por ello, para los años 2009-2019, $T P$ no compite con las publicaciones de Arqueología, que Clarivate Analytics considera solamente en Humanidades. Dentro de SSCI, la revista se incluyó en la categoría Historia entre 2009 y 2012, donde estuvo en el Q2 para los años 2009, 2011 y 2012. Pasó a Antropología desde 2013, donde se ha mantenido en el Q3 salvo en 2017.

Los datos de factor de impacto se han incrementado notablemente desde 2018, tanto en el cálculo del ranking tradicional para 2 años como para su cálculo alternativo para 5 años (Fig. 2).

\subsection{Datos de $T P$ en los indicadores elaborados a partir de Scopus}

El índice de citas alternativo a Web of Science es la base de datos Scopus, en la que también está indexada $T P$.

Trab. Prehist., 78, N. ${ }^{\circ} 2$, julio-diciembre 2021, pp. 207-216, ISSN: 0082-5638

https://doi.org/10.3989/tp.2021.12272 


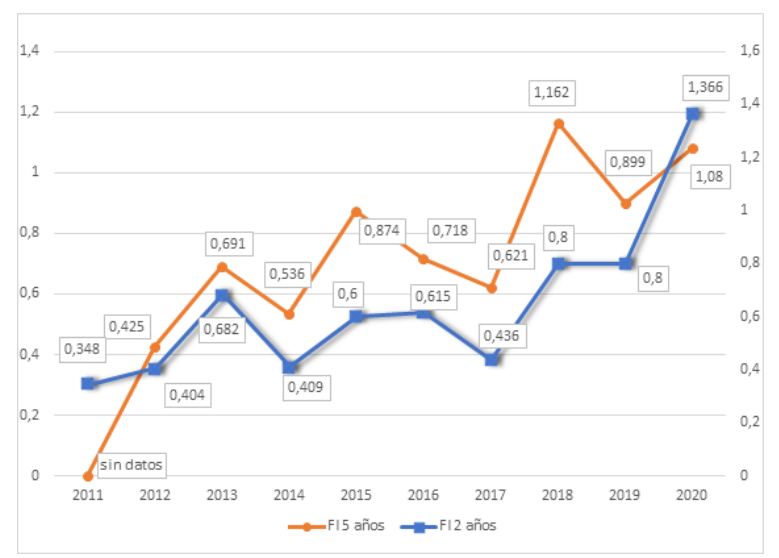

Fig. 2. Evolución del factor de impacto de Trabajos de Prehistoria, calculado sobre 2 y 5 años, en Journals Citation Reports (Web of Science) desde 2011 (en color en la versión electrónica).

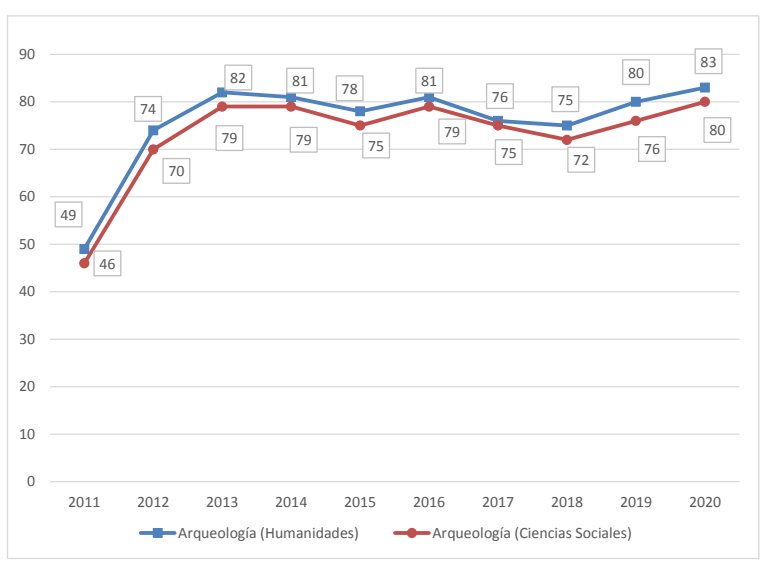

Fig. 3. Datos de Trabajos de Prehistoria en el periodo 2011-2020 en el indicador CiteScore de Scopus, expresado en percentiles (en color en la versión electrónica).

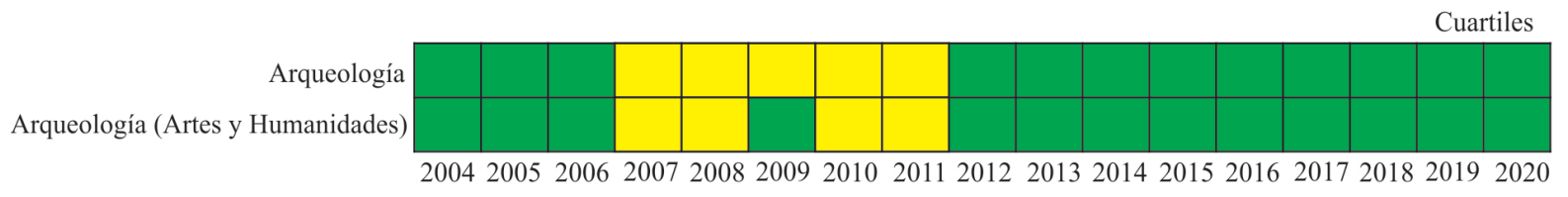

Fig. 4. Evolución de la posición de Trabajos de Prehistoria en los cuartiles (Q) de SCImago Journal Rank desde 2004. El tono más oscuro indica Q1, el más claro Q2 (en color verde y amarillo en la versión electrónica).

En este caso la revista se encuadra dentro de la categoría Arqueología, que cuenta con dos agrupaciones, una dentro de Ciencias Sociales y otra en Humanidades, con escasas diferencias en los títulos agrupados en cada una. El propio productor de Scopus, la editorial Elsevier B. V., publica un ranking de publicaciones basado en el indicador CiteScore, que se calcula por el porcentaje de citas por artículos publicados en un periodo de 4 años.

Los últimos datos publicados corresponden a 2020 (Tab. 1), en donde el CiteScore de TP es 1,6 (118 citaciones recibidas en 2017-2020 a 74 documentos publicados en el mismo periodo). Gracias a ello, la revista se sitúa en el Q1 en ambas agrupaciones de la categoría Arqueología. Elsevier aporta el dato en percentiles, estando representado el primer cuartil por las posiciones 75 a 99. TP se encuentra en este indicador en una notable posición, en el percentil 83 (puesto 49 entre 295 títulos) de las revistas de Arqueología en Humanidades y en el 80 (53 de 295) de las de Ciencias Sociales.

Respecto a los datos obtenidos en años anteriores, $T P$ se ha asentado en el Q1 en CiteScore desde 2013 (Fig. 3). El valor absoluto de la división de citas recibidas por artículos publicados en el mismo periodo de 4 años se sitúa por encima de 1 en los 8 últimos $(1,0$ en $2015 ; 1,1$ en 2014, 2017 y 2018; 1,2 en 2013; 1,3 en 2016 y 2019; 1,6 en 2020). La previsión para 2021 según los datos acumulados en septiembre es que se sitúe ya en 1,8 .
Los datos de Scopus son fuente para otros indicadores y rankings de revistas. El grupo SCImago presenta anualmente los resultados de SCImago Journal Rank $(S J R)$. Este índicador, mediante un algoritmo, pondera las citas recibidas según la posición respectiva de las revistas citantes. Al tomar los datos de Scopus se utiliza la misma clasificación de las revistas, por lo que $T P$ queda igualmente dentro de la categoría de Arqueología en Humanidades y en Ciencias Sociales.

En los últimos años $T P$ ha mejorado notablemente su posición en este ranking internacional de revistas. Los datos de 2020 actualizados en junio de 2021 del indicador $S J R$ se calculan a partir de las citas recibidas en 2020 por los artículos publicados en 2017-2019 en las publicaciones recogidas por la base de datos Scopus. TP se encuentra en el Q1, en la posición 18 entre 318 revistas de Arqueología-Humanidades y la 17 entre 301 de Arqueología-Ciencias Sociales. En ambos casos es la primera entre las revistas españolas. En el ámbito internacional es la primera publicación del ranking que se difunde en acceso abierto.

En el ranking de SCImago, TP se ha mantenido en el Q1 de las revistas de Arqueología desde 2012 (Figs. 4 y 5).

\footnotetext{
${ }^{3}$ Los datos de la revista en el portal SCImago Journal \& Country Rank pueden consultarse en esta dirección web: https://www.scimagojr.com/journalsearch.php?q=17500154724\&tip=sid
} 


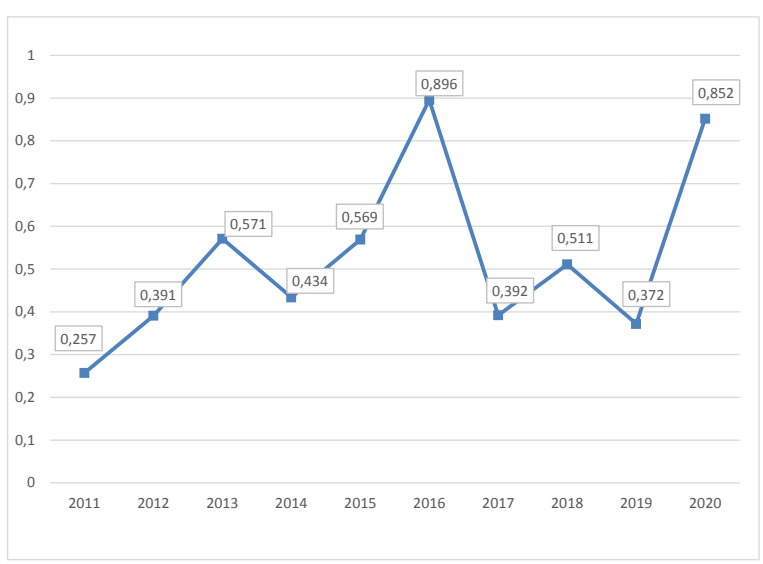

Fig. 5. Evolución de Trabajos de Prehistoria en el indicador SCImago Journal Rank desde 2011.

Cada vez más, la mayor parte de las citas proceden de otros títulos externos a la propia publicación (Fig. 6). En 2020 no solo ha aumentado el número de citas recibidas, sino que también mejora el porcentaje de artículos citados que ya supera al de los no-citados (Fig. 7). Esto indica que el mayor impacto de la revista no se debe a la especial difusión de artículos concretos sino a la buena recepción de la mayoría de sus contribuciones.

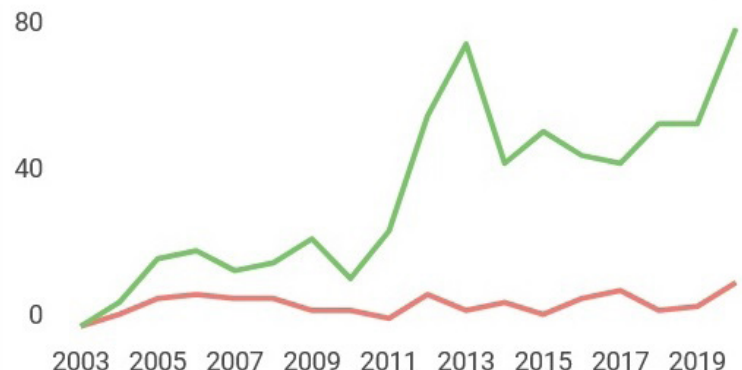

Fig. 6. Evolución desde 2003 del número de citas y autocitas a los artículos de Trabajos de Prehistoria recogidas en Scopus y calculada por SCImago. La línea superior corresponde al total de citas y la inferior a las autocitas (en color verde y rojo en la versión electrónica).

Scopus también es la base de los indicadores ofrecidos por el Centre for Science and Technology Studies (CWTS) de la Universidad de Leiden ${ }^{4}$. En el indicador Source Normalized Impact per Publication (SNIP), TP obtiene un valor de 0,99 en 2020. Este indicador se calcula mediante un algoritmo que pondera los resultados en función del patrón de citas recibidas por el conjunto de publicaciones de la categoría temática de la revista.

\footnotetext{
${ }^{4}$ Estos indicadores pueden consultarse en la dirección web: https://www.journalindicators.com/
}

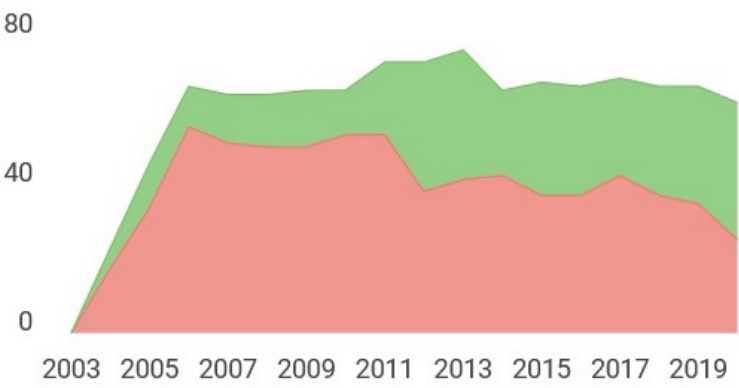

Fig. 7. Evolución desde 2003 del número de documentos citados y no citados entre los artículos de Trabajos de Prehistoria recogidos en Scopus y calculado por SCImago. La superficie superior corresponde al total de documentos citados y la inferior a los no citados (en color verde y rosado en la versión electrónica).

La tendencia de $T P$ en este caso es ligeramente positiva (Fig. 8), alcanzando su máximo valor (1,31) en 2018.

Otro indicador aportado por el CWTS es el Impact per Publication (IPP), que es el cálculo simple de las citas recibidas en un año determinado a los artículos publicados por una revista en los 3 años anteriores. El valor de $T P$ en 2020 es de 1,27, un caso claro de crecimiento respecto a años anteriores (Fig. 8). Por último, este centro ofrece también un gráfico sobre la evolución de las autocitas entre artículos de la propia revista (Fig. 9), en donde $T P$ se mantiene por debajo del $20 \%$ en los últimos años ${ }^{5}$.

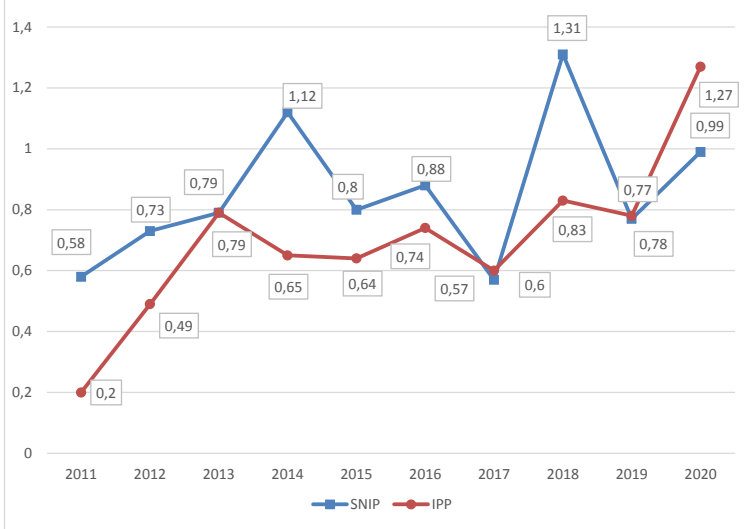

Fig. 8. Evolución de Trabajos de Prehistoria desde 2011 en los indicadores: Source Normalized Impact per Publication (SNIP) e Impact per Publication (IPP) elaborados por el Centre for Science and Technology Studies (CWTS) de la Universidad de Leiden (en color en la versión electrónica).

\footnotetext{
5 En este contexto se denomina autocitas a las citas recibidas desde artículos de la misma revista. Estos datos pueden excluirse en la elaboración de los indicadores bibliométricos o bien quedan integrados y se informa sobre su porcentaje como medida de precaución frente a posibles fraudes. No existe un porcentaje mínimo que deba cumplirse, pero puede considerarse que por debajo de un $20 \%$ tendría una incidencia mínima si se excluyeran del cálculo. Debe tenerse en cuenta que en Scopus se
} 


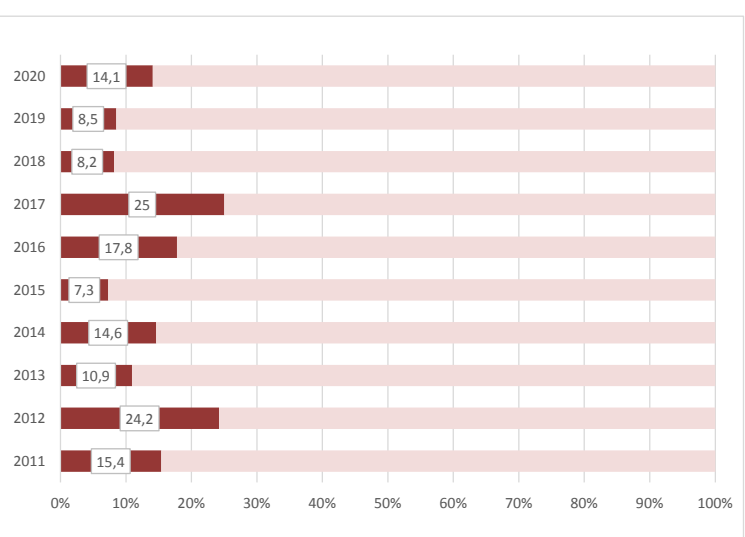

Fig. 9. Evolución del porcentaje de autocitas en Trabajos de Prehistoria desde 2011, calculado por el Centre for Science and Technology Studies (CWTS) de la Universidad de Leiden.
Revistas (IDR) tiene en cuenta las citas recibidas en un año a los artículos publicados en los cinco anteriores en una selección de revistas fuente recogidas en este portal bibliográfico. Actualmente este portal cubre el periodo de 2016 a 2020.

\begin{tabular}{|l|l|}
\hline $\begin{array}{c}\text { Fuente } \\
\text { de datos }\end{array}$ & \multicolumn{1}{|c|}{ Indicadores y posición en rankings 2020 } \\
\hline $\begin{array}{l}\text { Dialnet/ } \\
\text { Métricas }\end{array}$ & $\begin{array}{l}\text { IDR Índice Dialnet de Revistas (5 años): 0,960 } \\
\text { Cuartil 1 en Arqueología y Prehistoria (\#2/53) } \\
\text { Cuartil 1 en Historia (\#3/299) }\end{array}$ \\
\hline REDIB & $\begin{array}{l}\text { Calificador global (5 años): 32,203 } \\
\text { Cuartil 1 en clasificación global (\#42/1199) } \\
\text { Cuartil 1 en Antropología (\#1/9) } \\
\text { Cuartil 1 en Arqueología (\#2/13) }\end{array}$ \\
\hline $\begin{array}{l}\text { Sello de } \\
\text { calidad } \\
\text { FECYT }\end{array}$ & $\begin{array}{l}\text { Puntuación (5 años): 75,17 } \\
\text { Cuartil 1 en Arqueología, Antropología y } \\
\text { Etnología (\#2/18) }\end{array}$ \\
\hline
\end{tabular}

Tab. 2. Resumen de datos de Trabajos de Prehistoria en los rankings vigentes de revistas españolas o iberoamericanas.

\begin{tabular}{|c|c|c|c|c|c|c|}
\hline \multirow{2}{*}{ Año } & \multirow{2}{*}{ Impacto } & \multirow{2}{*}{ N. ${ }^{\circ}$ citas } & \multicolumn{2}{|c|}{ Arqueología y Prehistoria } & \multicolumn{2}{|c|}{ Historia } \\
\hline & & & Posición & Cuartil & Posición & Cuartil \\
\hline 2020 & 0,960 & 96 & $2 / 53$ & $\mathrm{C} 1$ & $3 / 299$ & $\mathrm{C} 1$ \\
\hline 2019 & 0,899 & 89 & $1 / 64$ & $\mathrm{C} 1$ & $1 / 313$ & $\mathrm{C} 1$ \\
\hline 2018 & 1,111 & 110 & $1 / 67$ & $\mathrm{C} 1$ & $1 / 315$ & $\mathrm{C} 1$ \\
\hline 2017 & 0,676 & 69 & $1 / 66$ & $\mathrm{C} 1$ & $1 / 311$ & $\mathrm{C} 1$ \\
\hline 2016 & 1,108 & 113 & $1 / 66$ & $\mathrm{C} 1$ & $1 / 312$ & $\mathrm{C} 1$ \\
\hline
\end{tabular}

Tab. 3. Evolución de Trabajos de Prehistoria desde 2016 en el indicador Índice Dialnet de Revistas (IDR) elaborado por Dialnet/Métricas.

\section{POSICIÓN DE TRABAJOS DE PREHISTORIA EN RANKINGS NACIONALES E IBEROAMERICANOS}

En España se han desarrollado otras iniciativas de rankings de revistas que pueden considerarse complementarias de los listados internacionales. Su cobertura se limita a publicaciones nacionales o bien del ámbito iberoamericano. En la tabla 2 se resumen los datos de $T P$ en 2020 en los principales indicadores que se elaboran a partir de estos productos.

En abril de 2021 la Fundación Dialnet ha completado las disciplinas de Humanidades en su portal de indicadores bibliométricos de revistas españolas e iberoamericanas: Dialnet/Métricas ${ }^{6}$. El Índice Dialnet de

refiere al porcentaje dentro de las fuentes incluidas en el propio índice, no contabiliza las posibles citas recibidas desde otras publicaciones.

${ }^{6}$ Los datos bibliométricos de TP en el portal Dialnet/Métricas pueden consultarse en la dirección web:

https://dialnet.unirioja.es/metricas/idr/revistas/1440
De acuerdo al $I D R$, TP se sitúa en el Q1 en dos categorías temáticas, Arqueología y Prehistoria e Historia (Tab. 3), ocupando las primeras posiciones en ambas disciplinas en todos los años analizados, entre más de 50 publicaciones de Arqueología y casi 300 de Historia. En este mismo portal se ofrecen una representación gráfica de la distribución de las citas dentro del intervalo analizado, así como datos de citas a nivel de artículo (Tab. 4).

Por su parte, el portal REDIB ofrece también su propio ranking de revistas ${ }^{7}$ elaborado por Clarivate Analytics a partir de las citas disponibles en Web of Science, de acuerdo a un algoritmo que pondera los resultados de citas recibidas en un año a los 5 anteriores, en función del patrón de citas de las categorías temáticas en las que se encuadra cada revista. Este recurso se limita a las publicaciones iberoamericanas de acceso abierto que se encuentre indexadas en ambas fuentes,

\footnotetext{
7 El ranking de REDIB puede consultarse en: https://redib.org/Ranking/Revistas
} 


\begin{tabular}{|c|c|c|c|}
\hline & Anualidad & Artículo & Citas \\
\hline 1 & 1993 & 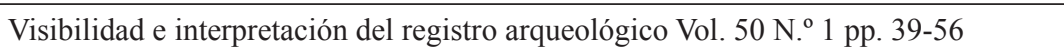 & 40 \\
\hline 2 & 2005 & $\begin{array}{l}\text { Ritual campaniforme, ritual colectivo: la necrópolis de cuevas artificiales de Valle } \\
\text { de las Higueras (Huecas, Toledo) Vol. } 62 \text { N. }{ }^{\circ} 2 \text { pp. } 67-90\end{array}$ & 26 \\
\hline 3 & 2003 & $\begin{array}{l}\text { Depósito funerario y recinto fortificado de la Edad del Bronce en el Castro del Chao } \\
\text { Samartín: argumentos para su datación Vol. } 60 \text { N. }{ }^{\circ} 2 \text { pp. } 143-151\end{array}$ & 24 \\
\hline 4 & 1998 & $\begin{array}{l}\text { Las comunidades campesinas en la cultura castreña } \\
\text { Vol. } 55 \mathrm{~N}^{\circ}{ }^{\circ} 2 \mathrm{pp} .127-150\end{array}$ & 23 \\
\hline 5 & 1989 & Megalitos, espacio y pensamiento Vol. 46 N. ${ }^{\circ} 1$ pp. $75-98$ & 23 \\
\hline 6 & 2003 & $\begin{array}{l}\text { Recintos de fosos del III milenio AC en la Meseta peninsular Vol. } 60 \text { N. }{ }^{\circ} 2 \text { pp. } 61 \text { - } \\
78\end{array}$ & 22 \\
\hline 7 & 2004 & $\begin{array}{l}\text { Reutilización de sepulcros colectivos en el Sureste de la Península Ibérica: la colec- } \\
\text { ción Siret Vol. } 61 \text { N. }^{\circ} 1 \text { pp. } 99-116\end{array}$ & 22 \\
\hline 8 & 1978 & $\begin{array}{l}\text { Una inhumación triple de facies Cogotas I en San Román de la Hornija (Valladolid) } \\
\text { Vol. } 35 \text { N. }^{\circ} 1 \text { pp. } 225-250\end{array}$ & 21 \\
\hline 9 & 1995 & $\begin{array}{l}\text { Arqueología y política el desarrollo de la arqueología española en su contexto histó- } \\
\text { rico Vol. } 52 \text { N N. }{ }^{\circ} 1 \text { pp. } 25-38\end{array}$ & 19 \\
\hline 10 & 1998 & $\begin{array}{l}\text { La monumentalización del Paisaje: percepción actual y sentido original en el Mega- } \\
\text { litismo de la Sierra de Barbanza (Galicia) Vol. } 55 \text { N. }{ }^{\circ} 1 \text { pp. } 63-80\end{array}$ & 19 \\
\hline
\end{tabular}

Tab. 4. Artículos publicados por Trabajos de Prehistoria que han recibido más citas desde 2011 según los datos recogidos en Dialnet/Métricas: Autores 1 y 5. Felipe Criado Boado; 2. Primitiva Bueno Ramírez, Rosa Barroso Bermejo y Rodrigo de Balbín Behrmann; 3. Ángel Villa Valdés y Luis Cabo Pérez; 4. M. Dolores Fernández-Posse y F. Javier Sánchez-Palencia; 6. Pedro Díaz del Río; 7. Alberto J. Lorrio e Ignacio Montero Ruiz; 8. Germán Delibes de Castro; 9. Margarita Díaz-Andreu y Gloria Mora; 10. Felipe Criado Boado y Victoria Villoch Vázquez.

\begin{tabular}{|c|c|c|c|c|c|c|}
\hline Año & Calificador global & $\mathbf{N}^{{ }^{\circ} \text { citas }}$ & $\begin{array}{c}\text { \% art. } \\
\text { citados }\end{array}$ & Posición global & Posición Arqueología & $\begin{array}{c}\text { Posición } \\
\text { Antropología }\end{array}$ \\
\hline 2020 & 32,203 & 127 & 26,02 & $\begin{array}{c}42 / 1199 \\
\text { Cuartil } 1\end{array}$ & $\begin{array}{c}2 / 13 \\
\text { Cuartil } 1\end{array}$ & $\begin{array}{c}1 / 9 \\
\text { Cuartil } 1\end{array}$ \\
\hline 2019 & 30,311 & 140 & 19,095 & $\begin{array}{c}49 / 1160 \\
\text { Cuartil } 1\end{array}$ & $\begin{array}{c}2 / 12 \\
\text { Cuartil } 1\end{array}$ & $\begin{array}{c}1 / 10 \\
\text { Cuartil } 1\end{array}$ \\
\hline 2018 & 26,380 & 87 & 16,746 & $\begin{array}{c}44 / 1038 \\
\text { Cuartil } 1\end{array}$ & $\begin{array}{c}1 / 13 \\
\text { Cuartil } 1\end{array}$ & $\begin{array}{c}1 / 11 \\
\text { Cuartil } 1\end{array}$ \\
\hline 2017 & 31,431 & 125 & 22,749 & $\begin{array}{c}17 / 947 \\
\text { Cuartil } 1\end{array}$ & $\begin{array}{c}1 / 7 \\
\text { Cuartil } 1\end{array}$ \\
\hline
\end{tabular}

Tab. 5. Posición de Trabajos de Prehistoria en el ranking REDIB desde 2017. Datos suministrados al portal REDIB por Clarivate Analytics.

REDIB y Web of Science, por lo que puede considerarse que es un ranking parcial. Dentro del mismo, $T P$ se encuentra en el Q1 de las categorías de Antropología y Arqueología en todos los años en los que se ha publicado (Tab. 5). En el total de las publicaciones, ocupa la posición 42 sobre 1199 títulos. REDIB ofrece también un gráfico de la distribución por años de las citas utilizadas en su cálculo (Fig. 10).

Finalmente, la Fundación Española para la Ciencia y la Tecnología (FECYT) ofrece un ranking de visibilidad e impacto de revistas científicas españolas de Humanidades y Ciencias Sociales que cuentan con su sello de calidad editorial ${ }^{8}$. Los datos publicados corresponden a los años 2018, 2019 y 2020, en donde $T P$ se mantiene en los primeros lugares dentro de la categoría conjunta de Arqueología, Antropología y Etnología (Tab. 6).

\section{CONCLUSIONES}

Los excelentes resultados de Trabajo de Prehistoria son un éxito colectivo cuyo primer protagonista es el

\footnotetext{
8 El ranking de FECYT puede consultarse en: https://calidadrevistas.fecyt.es/ranking
} 


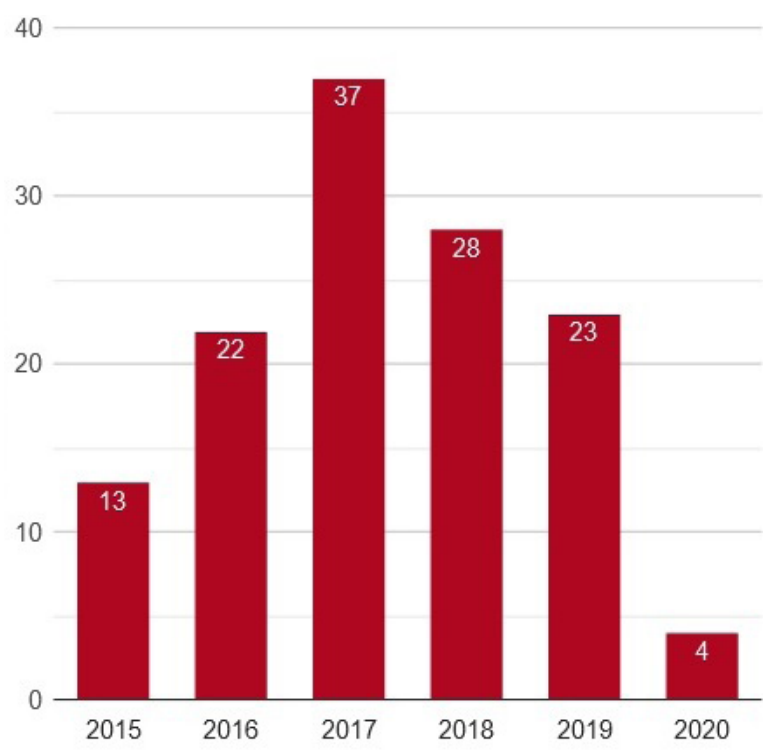

Fig. 10. Distribución por años de las 127 citas recibidas en 2020 por los artículos publicados en 2015-2020 por Trabajos de Prehistoria, según los datos recogidos en el ranking REDIB (Red Iberoamericana de Innovación y Conocimiento Científico, CSIC y Universia).

Consejo Superior de Investigaciones Científicas (CSIC). Desde su creación el objetivo de sus equipos directivos ha sido situarlo como organismo español de referencia, siendo su unidad editorial un agente fundamental de esa política. En la actualidad la Agencia Estatal CSIC es la $7^{\mathrm{a}}$ institución pública mundial de investigación mientras que Editorial $\mathrm{CSIC}^{9}$ ocupa la novena posición en el ranking mundial Scholarly Publishers Indicators (SPI) en Humanidades y Ciencias Sociales entre las editoriales mejor valoradas por expertos ${ }^{10}$.

A lo largo de esta trayectoria las revistas han modificado su papel fundacional. Sus principales fines han dejado de ser la creación y acrecentamiento de las bibliotecas a través de los intercambios y la difusión de la investigación realizada por los propios miembros de la institución. Las revistas de Editorial CSIC son a día de hoy una de las claves del prestigio internacional de la Agencia. Ello se debe en gran parte a que su política de acceso abierto lo es en un doble sentido: gratuidad e invitación a científicos de centros públicos y privados en todo el mundo a publicar los resultados de su investigación.

La calidad de la revista es el factor decisivo para aceptar esta invitación. En ella intervienen aspectos como la producción, distribución, cumplimiento de la periodicidad y, sobre todo, los contenidos. La buena aceptación de TP está directamente relacionada con el impacto que ha tenido la profesionalización e internacionalización de la Prehistoria como disciplina científica a escala mundial, en la investigación prehistórica de la

9 https://www.csic.es/es/ciencia-y-sociedad/editorial-csic
${ }^{10} \mathrm{http} / / /$ ilia.cchs.csic.es/SPI/prestigio_expertos_2018.php

\begin{tabular}{|c|c|c|c|c|}
\hline Año & Posición & Revista & Cuartil & Puntuación \\
\hline \multirow{4}{*}{2018} & 1 & Trabajos de Prehistoria & $\mathrm{C} 1$ & 99,49 \\
\cline { 2 - 5 } & 2 & Zephyrus & $\mathrm{C} 1$ & 72,94 \\
\cline { 2 - 5 } & 3 & Disparidades. Revista de Antropología & $\mathrm{C} 1$ & 62,38 \\
\cline { 2 - 5 } & 4 & AIBR. Revista de Antropología Iberoamericana & $\mathrm{C} 1$ & 58,47 \\
\cline { 2 - 5 } & 5 & Pasos. Revista de Turismo y Patrimonio Cultural & $\mathrm{C} 2$ & 58,21 \\
\hline \multirow{4}{*}{2019} & 1 & Pasos. Revista de Turismo y Patrimonio Cultural & $\mathrm{C} 1$ & 83,47 \\
\cline { 2 - 5 } & 2 & Trabajos de Prehistoria & $\mathrm{C} 1$ & 78,79 \\
\cline { 2 - 5 } & 3 & Disparidades. Revista de Antropología & $\mathrm{C} 1$ & 68,03 \\
\cline { 2 - 5 } & 4 & Virtual Archaeology Review & $\mathrm{C} 2$ & 66,85 \\
\hline \multirow{4}{*}{2020} & 5 & AIBR. Revista de Antropología Iberoamericana & 61,66 \\
\cline { 2 - 5 } & 1 & Pasos. Revista de Turismo y Patrimonio Cultural & $\mathrm{C} 1$ & 82,97 \\
\cline { 2 - 5 } & 2 & Trabajos de Prehistoria & $\mathrm{C} 1$ & 75,17 \\
\cline { 2 - 5 } & 3 & Virtual Archaeology Review & $\mathrm{C} 1$ & 74,05 \\
\cline { 2 - 5 } & 4 & AIBR. Revista de Antropología Iberoamericana & 62,63 \\
\hline
\end{tabular}

Tab. 6. Posición de Trabajos de Prehistoria en la categoría de Arqueología, Antropología y Etnología dentro del ranking de visibilidad e impacto de revistas científicas españolas de Humanidades y Ciencias Sociales con Sello de Calidad FECYT (Fundación Española para la Ciencia y la Tecnología, Ministerio de Ciencia, Innovación y Universidades, Gobierno de España). 
península ibérica y en sus practicantes. Desde los años de la renovación teórica promovida por la generación que accedió a puestos académicos en los 70 y 80 del siglo pasado la investigación peninsular ha llegado a niveles de excelencia e internacionalización comparables a sus pares europeos. Ello se debe al esfuerzo público, que ha diseñado y financiado la creación de infraestructuras e instituciones reguladoras y de evaluación. Pero sin duda también gracias al esfuerzo personal de todos aquellos que forman nuestra comunidad científica por desarrollar investigaciones de excelencia publicadas en revistas internacionales del más alto impacto.

$T P$ se beneficia de esta dinámica publicando excelentes contenidos. El reconocimiento de su originalidad y relevancia se debe al trabajo de los comités editoriales y a la colaboración y el esfuerzo anónimo y desinteresado de nuestras evaluadoras y evaluadores. Su criterio y experiencia mantienen el elevado estándar de calidad que ha caracterizado históricamente la trayectoria de la revista.

Los resultados obtenidos por $T P$ en los sistemas internacionales son muy satisfactorios a pesar de la feroz competencia de las grandes multinacionales del sector editorial. Estos resultados se mantienen incluso teniendo en cuenta el muy específico nicho de la revista, que publica contenidos predominantemente en español y resultados de investigación de interés mayoritariamente regional. Cualquier revista que no se edite exclusivamente en inglés tiene grandes dificultades para alcanzar los niveles de citación necesarios para acceder a la élite de un mercado global como es el de las publicaciones científicas (Di Bitetti y Ferreras 2017). Los dos principales índices de citas internacionales, Scopus y Web of Science, cubren publicaciones en diferentes idiomas, pero sin duda mantienen un importante sesgo hacia las fuentes en inglés (Vera et al. 2019). Todas estas circunstancias subrayan aún más la posición consolidada de Trabajos de Prehistoria como la revista española de referencia en su campo.

\section{AGRADECIMIENTOS}

Destacamos la dedicación y profesionalidad del Departamento de Edición Electrónica de la Editorial CSIC. En esta ocasión, Juan Ignacio Pérez-Alcalde y Daniel Bote nos facilitaron la imagen base para la elaboración de la figura 1 .

\section{BIBLIOGRAFÍA}

Cagan, R. 2013: "The San Francisco Declaration on Research Assessment". Disease Models \& Mechanisms 6: 869-870. https://doi.org/10.1242/dmm.012955

Delgado López-Cózar, E.; Ràfols, I. y Abadal, E. 2021: “Letter: A call for a radical change in research evaluation in Spain = Carta: Por un cambio radical en la evaluación de la investigación en España”. Profesional de la información 30 (3): e300309. https://doi.org/10.3145/epi.2021.may.09

Di Bitetti, M. S. y Ferreras, J. A. 2017: "Publish (in English) or perish: The effect on citation rate of using languages other than English in scientific publications". Ambio 46: 121-127. https://doi.org/10.1007/s13280-016-0820-7

Hicks, D.; Wouters, P.; Waltman, L.; De Rijcke, S. y Ràfols, I. 2015: "Bibliometrics: The Leiden Manifesto for research metrics". Nature 520: 429-431. https://doi.org/10.1038/520429a

Rodríguez Alcalde, Á; San Millán Bujanda, M. J.; Sánchez Nistal, J.

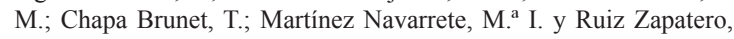
G. 1993: "Análisis bibliométrico de Trabajos de Prehistoria: un chequeo a la prehistoria española de las tres últimas décadas". Trabajos de Prehistoria 50: 11-37. https://doi.org/10.3989/tp.1993.v50.i0.487

Rodríguez Yunta, L. 2014: "Indización en profundidad y aplicación de metadatos a materiales suplementarios en la edición de revistas". Anuario ThinkEPI 8: 207-210. http://hdl.handle.net/10261/98846

Rodríguez Yunta, L.; Vidal Liy, J. I. y Martínez Navarrete, M. ${ }^{\text {a }}$ I. 2019: "Análisis bibliométrico de la revista Trabajos de Prehistoria en el contexto de las revistas españolas de Arqueología y Prehistoria recogidas por Scopus e ÍnDICEs CSIC'. Trabajos de Prehistoria 76 (2): 199-218. https://doi.org/10.3989/tp.2019.12233

Vera Baceta, M. A.; Thelwall, M. y Kousha, K. 2019: "Web of Science and Scopus language coverage". Scientometrics 121: 1803-1813. https://doi.org/10.1007/s11192-019-03264-z

Walters, W. H. 2017: "Citation-Based Journal Rankings: Key Questions, Metrics, and Data Sources". IEEE Access 5: 22036-22053. https://doi.org/10.1109/ACCESS.2017.2761400. 